\title{
Spontaneous tumor lysis syndrome in small cell lung cancer
}

\author{
Ryan K. Dean, D0, Rogin Subedi, MBBS, and Mijung Lee, MD \\ Department of Medicine, State University of New York Upstate Medical University, Syracuse, New York
}

\section{ABSTRACT}

Tumor lysis syndrome is a set of metabolic disturbances that can be seen during the destruction of tumor cells and is an oncologic and metabolic emergency. The syndrome is rare in those with solid tumors, and even more rare in those with solid tumors who have not yet received chemotherapy. We present a case of tumor lysis syndrome in a patient with small cell lung cancer.

KEYWORDS Oncologic emergency; small cell lung cancer; spontaneous tumor lysis syndrome

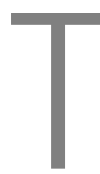
umor lysis syndrome (TLS), a constellation of metabolic and electrolyte disturbances that can be seen during the destruction of tumor cells, is an oncologic and metabolic emergency. It is most commonly seen in tumors with high cell turnover and growth rates (i.e., hematologic malignancies) and in bulky tumors that are highly sensitive to chemotherapeutic agents. TLS is rarely seen in those with solid tumors who have not yet received chemotherapy. Here we present a case of spontaneous TLS in a patient with a new diagnosis of small cell lung cancer.

\section{CASE STUDY}

A 76-year-old woman with T4 N3 M1c small cell lung cancer with metastases to the liver, diagnosed 5 days earlier, initially presented to the outpatient clinic to start chemotherapy. Her physical status had rapidly worsened in the preceding days, including lower-extremity edema, fatigue, and dyspnea, and she was sent to the emergency department for further evaluation. A chest radiograph showed a large right pleural effusion with a right hilar mass. Her serum potassium was $6.2 \mathrm{mmol} / \mathrm{L}$; bicarbonate, $14 \mathrm{mmol} / \mathrm{L}$; blood urea nitrogen, $110 \mathrm{mg} / \mathrm{dL}$; creatinine, $4.1 \mathrm{mg} / \mathrm{dL}$ (baseline 1.3); phosphorous, $7.1 \mathrm{mg} / \mathrm{dL}$; ionized calcium, $1.07 \mathrm{mmol} / \mathrm{L}$; lactic acid, $6.1 \mathrm{mmol} / \mathrm{L}$; and uric acid, $21.7 \mathrm{mg} / \mathrm{dL}$. The patient was given rasburicase and started on intravenous fluids with sodium bicarbonate. She received a total of two doses of rasburicase with a decrease in her uric acid to $3.8 \mathrm{mg} / \mathrm{dL}$. Her lactic acidosis continued to increase. Due to her rapidly declining condition, the patient died shortly thereafter.

\section{DISCUSSION}

TLS is characterized by massive disruption of tumor cells with release of intracellular contents into the blood, causing severe metabolic derangements, including hyperkalemia, hyperphosphatemia, hyperuricemia, hypocalcemia, and metabolic acidosis. The release of these intracellular contents can progress to clinically toxic effects and potentially fatal complications, including acute kidney injury, seizures, cardiac arrhythmias, or death. In the current Cairo and Bishop classification system, TLS may be labeled as laboratory or clinical. ${ }^{1,2}$ Laboratory TLS requires two or more of the following metabolic abnormalities to occur within 3 days before or up to 7 days after initiation of therapy: hyperuricemia, hyperkalemia, hyperphosphatemia, and hypocalcemia (Table 1). Clinical TLS is present when laboratory TLS is associated with an increased creatinine level, seizures, cardiac dysrhythmias, or sudden death. ${ }^{1,2}$

The incidence and severity of TLS depend on several factors and the characteristics of the malignancy, such as cancer mass, potential for lysis of the tumor cells, and patient characteristics. Cancers with high potential for cell lysis include hematologic malignancies, such as high-grade lymphomas, acute leukemias, and other rapidly proliferating tumors. In addition, the greater the cancer burden, the greater the quantity of cellular contents released once lysed and the greater the potential for TLS. ${ }^{2}$

While TLS is well established in hematologic malignancies, the prevalence of TLS associated with solid tumors, while it may be higher than previously thought, still remains relatively rare, with much evidence based primarily on case reports. ${ }^{3,4}$ To this end, spontaneous TLS in solid tumors is an especially rare occurrence and has been reported in the literature in only a few instances. The first reported case of spontaneous TLS in small cell lung cancer was in 2008, and since that time five other cases have been reported. ${ }^{3,5-8}$ TLS is generally not seen in solid tumors because they have such a low proliferative index. However, small cell lung cancer varies from most solid

Corresponding author: Ryan K. Dean, DO, Department of Medicine, State University of New York Upstate Medical University, 750 East Adams Street, Syracuse, NY 13210 (e-mail: deanr@upstate.edu) 
Table 1. Criteria for laboratory tumor lysis ${ }^{\star}$

\begin{tabular}{ll}
\hline Test & \multicolumn{1}{c}{ Criteria } \\
\hline Uric acid & $\geq 476 \mathrm{umol} / \mathrm{L}$ or $>25 \%$ increase from baseline \\
Potassium & $\geq 6 \mathrm{mmol} / \mathrm{L}$ or $>25 \%$ increase from baseline \\
Phosphorus & $\geq 2.1 \mathrm{mmol} / \mathrm{L}$ in children or $\geq 1.45 \mathrm{mmol} / \mathrm{L}$ in adults or $>25 \%$ \\
& increase from baseline \\
Calcium & $\leq 1.75 \mathrm{mmol} / \mathrm{L}$ or $>25 \%$ decrease from baseline \\
\hline
\end{tabular}

*Laboratory tumor lysis syndrome is defined as $\geq 2$ of these laboratory findings within 3 days before or 7 days after initiation of chemotherapy.

tumors in that it has a higher rate of cell turnover and may present with a higher degree of tumor burden. One specific distinction has been described between spontaneous TLS and posttreatment TLS based on the magnitude of hyperphosphatemia. Spontaneous TLS is thought to have lower levels of phosphorous due to its reutilization in the synthesis of new tumor cells, whereas posttreatment TLS has higher levels of phosphorous due primarily to cell destruction. ${ }^{2}$ Despite being less commonly seen, TLS from solid tumors appears to carry a higher mortality rate than TLS in hematologic malignancies, with the mortality reported as high as $40 \%{ }^{2,9}$ There is no evidence comparing mortality rates of spontaneous TLS in solid tumors due to its relative dearth in the literature.

Although TLS, and particularly spontaneous TLS, is rare in solid tumors, it is important for the clinician to be aware of this phenomenon, as prompt recognition and treatment can prevent significant morbidity and mortality.

1. Cairo MS, Bishop M. Tumour lysis syndrome: new therapeutic strategies and classification. Br J Haematol. 2004;127(1):3-11. doi:10.1111/j.13652141.2004.05094.x. PMID:15384972.

2. Howard SC, Jones DP, Pui CH. The tumor lysis syndrome. $N$ Engl J Med. 2011;364(19):1844-1854. doi:10.1056/NEJMra0904569. PMID:21561350.

3. Jaiswal A, Vaidya G, Baral D, Lee M. A rare case of spontaneous tumor lysis syndrome in small cell lung cancer. Chest. 2015;148(4 Suppl):569A,569B.

4. Kekre N, Djordjevic B, Touchie C. Spontaneous tumor lysis syndrome. CMAJ. 2012;184(8):913-916. doi:10.1503/cmaj.111251. PMID:22496380.

5. Huet J, Essig R. Initial report of spontaneous tumor lysis syndrome in small cell lung cancer. Chest. 2008;134(4 Suppl 2):56C. doi:10.1378/ chest.134.4_MeetingAbstracts.c56002.

6. Padhi P, Singh S. Spontaneous tumor lysis syndrome in a patient with metastatic small cell carcinoma of the lung. J Cancer Sci Ther. 2012;4 (6):164-166. doi:10.4172/1948-5956.1000133.

7. Martínez-Sáez O, Mezquita L, Caravaca-Fontan F, et al. Spontaneous tumor lysis syndrome in the setting of small cell lung cancer: report of two cases and review of the literature. Cancer Treat Res Comm. 2016;9:92-95. doi:10.1016/j.ctarc.2016.08.005.

8. Jallad B, Hamdi T, Latta S, Alhosaini MN, Kheir F, Iroegbu N. Tumor lysis syndrome in small cell lung cancer: a case report and review of the literature. Onkologie. 2011;34(3):129-131. doi:10.1159/000324791. PMID:21358219.

9. Okamoto K, Kinoshita T, Shimizu M, et al. A case of spontaneous tumor lysis syndrome in a patient with ovarian cancer. Case Rep Obstet Gynecol. 2015;2015:461870. PMID:26161277. 